\title{
ANALYTIC PROPERTIES OF THE RETURN MAPPING OF LIÉNARD EQUATIONS
}

\author{
J. P. FranÇOISE
}

\section{Introduction}

Bautin's approach to the bifurcation theory of limit cycles has been recently generalized in the framework of complex analysis ([8],[9]). There are now more cases where the Bautin ideal is known. A systematic study of the Poincaré center focus problem via Abel equations entailed several new examples of Bautin ideals $([2],[3])$.

This article deals with Liénard equations which have been used in many applications (c.f. [10]). Liénard equations play certainly a key role in Hilbert's $16^{\text {th }}$ problem, as suggested for instance in ([19],[20]), because of the topological simplicity of the return mapping. Limit cycles encircle the origin and are necessarily contained in the domain of existence of the return mapping. This domain of existence may of course not be equal to the domain of convergence of the analytic series which defines the return mapping in a neighborhood of the origin. Nevertheless it is interesting to produce an estimate of the size of this domain of convergence.

Many contributions have been previously done to Liénard equations and particularly by N. G. Lloyd and his co-workers ([1],[5],[14],[15],[16],[17]). But the approach via the Lyapunoff series which is used in all these references does not entail information on the domain of convergence of the first return mapping itself.

We develop here a direct computation of the return mapping (indeed of its converse) which yields an estimate of the domain of convergence by a majorizing series techniques and a recurrency relation for the coefficients which entail the Bautin ideal.

Application of the complex analysis methods of $([8],[9])$ is then straightforward. In Liénard case, the use of the Hironaka polynomial division theorem is replaced by a very simple and explicit argument. This displays a bound to the number of complex limit cycles in a fixed neighborhood of the origin.

Examples produced with perturbation theory (first-order) of Hamiltonians by A. Lins Neto, W. de Melo and C. C. Pugh ([12]) show that this bound is optimal (recall that the complex bound is at least twice the real bound).

Received September 22, 2000.

This research was made possible under a grant (FREMSU) of the Ministère de l'Education et de la Recherche allowed to the GSIB.. 
To end this introduction we mention other recent references to generalized Liénard equations $([6],[7],[11],[13],[18])$ to which eventually the same type of analysis should apply.

\section{Computation of the Bautin ideal}

The perturbation of the linear part into a focus adds one real limit cycle to the number obtained when the linear part is a center. We consider only the case where the linear part is a center to simplify the computations.

This paragraph is concerned with the polynomial vector fields $X$ of the following type:

$$
X=-x \partial / \partial y+y \partial / \partial x+\sum_{i=1}^{d}\left[\lambda_{i} x^{i}\right] y \partial / \partial y .
$$

and the associated flow, solution of the system:

$$
\begin{gathered}
\dot{x}=y \\
\dot{y}=-x+\sum_{i=1}^{d}\left[\lambda_{i} x^{i}\right] y=-x+p(x) y .
\end{gathered}
$$

The equations of the flow yield a second order differential equation classically named the Liénard equation. Note that this differential system is sometimes written:

$$
\begin{gathered}
\dot{x}=Y-P(x), \\
\dot{Y}=-x,
\end{gathered}
$$

which is easily changed into the preceding ones with $y=Y-P(x)$, if $P^{\prime}(x)=$ $-p(x)$.

Write (1.2) in polar coordinates $(r, \theta)$ :

$$
x=r \cos \theta, \quad y=r \sin \theta .
$$

This displays:

$$
\begin{gathered}
2 r \dot{r}=2(x \dot{x}+y \dot{y}), \quad r \dot{r}=r^{2} p(r \cos \theta) \sin ^{2} \theta \\
\dot{\theta}=(x \dot{y}-y \dot{x}) /\left(x^{2}+y^{2}\right)=-1+\sin \theta \cos \theta p(r \cos \theta) .
\end{gathered}
$$

This yields:

$$
d r / d \theta=r p(r \cos \theta) \sin ^{2} \theta /[-1+\sin \theta \cos \theta p(r \cos \theta)] .
$$

Bautin's approach is based on the study of solutions of (1.6) $r=r(\theta)$ so that $r(0)=r_{0}$, given as an expansion:

$$
r_{0}=r+v_{2}(\theta) r^{2}+\ldots+v_{k}(\theta) r^{k}+\ldots
$$


Comparison between (1.6) and (1.7) yields:

$$
\begin{aligned}
& \sum_{k \geq 1} v_{k}^{\prime}(\theta) r^{k}[-1+\sin \theta \cos \theta p(r \cos \theta)] \\
&+\sum_{k \geq 1}\left[k v_{k}(\theta) p(r \cos \theta) \sin ^{2} \theta\right] r^{k}=0 .
\end{aligned}
$$

This displays the following recurrency relation on the coefficients $v_{k}(\theta)$ :

$$
v_{k}^{\prime}(\theta)=\sum_{l=1}^{d} \lambda_{l}(\cos \theta)^{l} \sin \theta\left[\cos \theta v_{k-l}^{\prime}(\theta)+(k-l) \sin \theta v_{k-l}(\theta)\right] .
$$

The main result of this paragraph is:

Theorem 1.1. Let $2 n$ be the largest even integer such that $2 n \leq d$. Define the Bautin ideal of the differential system (1.1) as the ideal generated by the coefficients of the return mapping (equivalently of its inverse) $v_{k}(2 \pi)$ (cf [2][3]). This Bautin ideal is generated by $\left(\lambda_{2}, \ldots, \lambda_{2 n}\right)$. Define the Bautin index $B$ as the first integer $k$ so that the increasing sequence of ideals generated by the $k$ first coefficients $v_{k}(2 \pi)$ becomes stationary (cf [2][3]). The Bautin index of (1.1) is equal to $2 n+1$.

The proof of the theorem displays several lemmas of independent interest.

Let $I$ be an ideal of $R\left[\lambda_{1}, \ldots, \lambda_{d}\right]$. It is convenient to denote $v_{k}(\theta) \in I$ to mean that for all values of $\theta, v_{k}(\theta)$ is a polynomial in the parameters $\left(\lambda_{1}, \ldots, \lambda_{d}\right)$ which belongs to the ideal $I$. In the following $c_{k}$ denotes a sequence of nonzero numbers (independent of the parameters) which will be defined inductively. Choose the initial conditions for the recurrency relation (1.9) as:

$$
v_{1}(\theta)=1 \text {. }
$$

Then (1.9) yields:

$$
v_{2}^{\prime}(\theta)=\lambda_{1} \cos \theta \sin ^{2} \theta, \text { thus : } v_{2}(\theta)=\lambda_{1} w_{2}^{1}(\sin \theta), w_{2}^{1}(0)=0 .
$$

This entails:

$$
v_{2}(2 \pi)=0 \text {. }
$$

The first coefficient which really matters for the Bautin ideal is the next one. The recurrency relation (1.9) displays:

$$
v_{3}^{\prime}(\theta)=\lambda_{1} \cos \theta \sin \theta\left[\cos \theta v_{2}^{\prime}+2 \sin \theta v_{2}\right]+\lambda_{2} \cos ^{2} \theta \sin ^{2} \theta .
$$

This yields:

$$
v_{3}(\theta)=f_{3}(\theta)+\lambda_{1} w_{3}^{1}(\sin \theta)
$$

with:

$$
w_{3}^{1}(0)=0
$$


and

$$
f_{3}(\theta) \in\left(\lambda_{2}\right)
$$

and

$$
v_{3}(2 \pi)=c_{3} \lambda_{2}, c_{3}=\int_{0}^{2 \pi} \cos ^{2} \theta \sin ^{2} \theta d \theta \neq 0 .
$$

We now prove by induction the following:

Lemma 1.2. Let $k_{0}$ be the maximal integer so that $2 k_{0} \leq k-1$ and $k_{1}$ be the maximal integer so that $2 k_{1}+2 \leq k$. The coefficient $v_{k}(\theta)$ displays the decomposition:

$$
v_{k}(\theta)=f_{k}(\theta)+\sum_{j=0}^{k_{1}} \lambda_{2 j+1} w_{k}^{2 j+1}(\sin \theta, \lambda)
$$

where:

$$
f_{k}(\theta) \in\left(\lambda_{2}, \ldots, \lambda_{2 k_{0}}\right),
$$

Proof. This is certainly true for $v_{2}(\theta)$ and $v_{3}(\theta)$. Assume this is so inductively. The recurrency relation (1.9) shows that the term $f_{k-l}$ contributes to the ideal $\left(\lambda_{2}, \ldots, \lambda_{2 k_{0}}\right)$ and the element $w_{k-l}^{2 j+1}(\sin \theta, \lambda)$ contributes either (for $l$ even) to an element of the ideal $\left(\lambda_{2}, \ldots, \lambda_{2 k_{0}}\right)$ or (for $l=2 h+1$ odd) produces:

$$
\begin{gathered}
\lambda_{2 h+1}(\cos \theta)^{2 h+1} \sin \theta\left[\cos ^{2} \theta \sum_{j} \lambda_{2 j+1} w_{k-l}^{2 j+1}(\sin \theta, \lambda)^{\prime}\right. \\
\left.+\sin \theta(k-2 h-1) \sum_{j} \lambda_{2 j+1} w_{k-l}^{2 j+1}(\sin \theta, \lambda)\right],
\end{gathered}
$$

which (once integrated against $\theta$ contributes to $\lambda_{2 h+1} w_{k}^{2 h+1}(\sin \theta, \lambda)$ ).

An immediate consequence of the proposition is the following:

Lemma 1.3. The coefficients $v_{k}(2 \pi)$ belong to the ideal $\left(\lambda_{2}, \ldots, \lambda_{2 k_{0}}\right)$.

Proof. The coefficient $w_{k}^{2 j+1}(\sin \theta, \lambda)$ vanishes for $\theta=2 \pi$, hence

$$
v_{k}(2 \pi)=f_{k}(\theta) \in\left(\lambda_{2}, \ldots, \lambda_{2 k_{0}}\right) .
$$

This last result can be improved for the coefficients $v_{k}(2 \pi)$ of odd order $k$. Denote $n=[d / 2]$ (integer part of $d / 2$ ). 
Lemma 1.4. For all odd values of $k=2 k_{0}+1\left(k_{0}=1, \ldots, n\right)$, the coefficient $v_{2 k_{0}+1}(2 \pi)$ is such that:

$$
v_{2 k_{0}+1}(2 \pi) \in\left(\lambda_{2}, \ldots, \lambda_{2 k_{0}-2}\right)+c_{2 k_{0}+1} \lambda_{2 k_{0}}
$$

with:

$$
c_{2 k_{0}+1}=\int_{0}^{2 \pi}(\cos \theta)^{2 k_{0}}(\sin \theta)^{2} d \theta \neq 0 .
$$

Proof. In the recurrency relation (1.9), the only term which contributes to $\lambda_{2 k_{0}}$ is:

$$
\lambda_{2 k_{0}}(\cos \theta)^{2 k_{0}} \sin \theta[\sin \theta],
$$

which yields (1.19).

We now conclude with the proof of the theorem 1.1.

We consider the increasing sequence of ideals generated by the coefficients:

$$
\left(v_{2}(2 \pi), \ldots, v_{k}(2 \pi)\right) \text {. }
$$

The first coefficient $v_{2}(2 \pi)$ is zero, hence the first one which contributes to the Bautin ideal is $v_{3}(2 \pi)$ which is proportional to $\lambda_{2}$. Consider now increasing values of the integer $k$. The sequence of ideals does not increase at a step $v_{k}(2 \pi)$ with even $k$. When $k$ is odd, $k=2 k_{0}+1, v_{2 k_{0}+1}$ contributes with a term $c_{2 k_{0}+1} \lambda_{2 k_{0}}$ with $c_{2 k_{0}+1} \neq 0$, hence the ideal strictly increases and becomes equal to:

$$
\left(\lambda_{2}, \ldots, \lambda_{2 k_{0}}\right) \text {. }
$$

This proceeds till:

$$
k_{0}=n=[d / 2],
$$

and latter the ideal does not increase any more. The Bautin ideal is thus equal to:

$$
\left(\lambda_{2}, \ldots, \lambda_{2 n}\right),
$$

and the increasing sequence of ideals (1.21) stabilizes at the step $2 k_{0}+1=2 n+1$. This ends the proof of the theorem.

The recurrency relation entails as well the following result:

Theorem 1.5. For all values of $\lambda$, there is a neighborhood of the origin on which the number of real limit cycles is less than $n-1$ ( $n$ if we consider the perturbation of a focus).

Proof. This is a consequence of the classical Bautin's argument. Collecting the terms of the first return mapping, we write the equation for the real limit cycles as:

$(1.24) v_{3}(2 \pi) r^{3}(1+\ldots)+v_{5}(2 \pi) r^{5}(1+\ldots)+\ldots+v_{2 n+1}(2 \pi) r^{2 n+1}(1+\ldots)=0$. 
Successive applications of Rolle's lemma show that the number of real positive zeroes of (1.24) is less than $n-1$. This shows that the maximal number of limit cycles which can bifurcate when the linear part is of focus type is $n$ (because it adds up one term in (1.24)). Note that this bound is in agreement with the bound foreseen in A. Lins neto-W. de Melo-C.C. Pugh conjecture. (cf [12]). Of course this computation does not entail any control of the size of the domain on which the number of limit cycles is less than $n$ in terms of the coefficients of the perturbation. This is the reason to develop further analysis based on the complexification of the return mapping. To conclude this paragraph, let us consider the famous example of the van der Pol equation:

$$
\dot{x}=y,
$$

$$
\dot{y}=-x+p(x) y, p(x)=\lambda\left(1-x^{2}\right),
$$

In that case, there is a perturbation of the linear part into a focus, $d=2$, and thus $n=1$ and we obtain a bound of 1 to the number of limit cycles. This is a well known result and it is also known that in van der Pol example there is effectively a limit cycle which is born from a supercritical Hopf bifurcation. Note that the existence of a subcritical Hopf bifurcation giving birth to two limit cycles needs a polynomial $p(x)$ of degree $d=4$. Such examples are for instance quite important for modeling in electrophysiology and biochemistry.

\section{Estimates of the radius of convergence of the first return mapping}

Let $f_{\lambda}(x)=\sum a_{k}(\lambda) x^{k}$ be an analytic series in $x$ with polynomial coefficients in the parameters $\lambda=\left(\lambda_{1}, \ldots, \lambda_{d}\right)$. Denote $\left|a_{k}\right|$ (norm of the polynomial $a_{k}$ ) as the sum of the absolute value of the coefficients and $|\lambda|=\left|\lambda_{1}\right|+\ldots+\left|\lambda_{d}\right|$. Recall now the following:

Definition 2.1. The series $f_{\lambda}$ is called an $A_{0}$-series if the following two conditions are satisfied:

There are positive constants $K_{1}, K_{2}, K_{3}, K_{4}$ such that:

$$
\begin{gathered}
\operatorname{deg}\left(a_{k}\right) \leq K_{1} k+K_{2}, \\
\left|a_{k}\right| \leq K_{3} K_{4}^{k} .
\end{gathered}
$$

$A_{0}$-series form a subring of the ring of formal power series in $x$ with polynomial coefficients in $\lambda$. All the usual analytic operations, like substitution to a given analytic function, composition, inversion,... transform $A_{0}$-series into themselves. $A_{0}$-series have been precisely introduced (in the subject) by M. Briskin and Y. Yomdin ([4]).

In the following, we also denote $f_{\lambda}$ the complex analytic function defined for all $\lambda \in C^{D}$ on a disc $D(0, R)$ by the $A_{0}$-series.

In the Liénard case, the following holds: 
Proposition 2.2. The series (1.7) is for all values of $\theta$ a $A_{0}$-series with:

$$
K_{1}=1, K_{2}=-1, K_{3}=\pi / 2, K_{4}=2 \text {, }
$$

if $|\lambda|<1$.

Proof. The inverse series of (1.7), which is indeed the Bautin series:

$$
r=r_{0}+w_{2}(\theta) r_{0}^{2}+\ldots+w_{k}(\theta) r_{0}^{k}+\ldots
$$

solves the differential equation (1.6). We use Siegel majorizing series techniques. A rough majorizing series (both in $\lambda$ and $r_{0}$ ) of (1.6) is provided by the solution $M$ of the differential equation:

$$
d M / d \theta=[M|\lambda| M /(1-M)] /[1-|\lambda| M /(1-M)],
$$

which displays the expansion:

$$
M=r_{0}+M_{2}(\theta) r_{0}^{2}+\ldots+M_{k}(\theta) r_{0}^{k}+\ldots
$$

The coefficients $M_{k}(\theta)$ are inductively defined by a recurrency relation which is of following type:

$$
M_{k}^{\prime}(\theta)=S_{k}\left[M_{2}(\theta), \ldots, M_{k-1}(\theta)\right], k \geq 2,
$$

where $S_{k}$ is a polynomial with positive coefficients.

Denote

$$
M_{k}=\operatorname{Max}\left[M_{k}(\theta), \theta \in[0,2 \pi]\right] .
$$

The equation (2.8) yields the following inequality:

$$
M_{k} \leq(2 \pi) S_{k}\left[M_{2}, \ldots, M_{k-1}\right], k \geq 2 .
$$

This displays a new majorizing series for $(2.5) W\left(r_{0}\right)$ solution of the algebraic equation:

$$
W\left(r_{0}\right)-r_{0}=(2 \pi)|\lambda| W\left(r_{0}\right)^{2} /\left[1-(1+|\lambda|) W\left(r_{0}\right)\right] .
$$

The algebraic equation (2.11a) has a unique analytic solution $W\left(r_{0}\right)$ which is tangent to $r_{0}$ for small values of $r_{0}$. This equation yields $r_{0}$ in terms of $W\left(r_{0}\right)$ :

$$
r_{0}=W\left(r_{0}\right)-(2 \pi)|\lambda| W\left(r_{0}\right)^{2} /\left[1-(1+|\lambda|) W\left(r_{0}\right)\right] .
$$

Elementary considerations on majorizing series show that the converse of a majorizing series provides a majorizing series for the converse. This immediately provides an estimate of the radius of convergence of (1.7):

$$
R(\lambda)=1 /[1+|\lambda|]
$$

and the proof of the proposition 2.2 . 
The estimate of the radius of convergence can be improved (for some values of the parameters) with an elementary scaling argument in the case of Liénard's equations. The proof given above works actually for an arbitrary polynomial perturbation of the rotation flow (with some changes of notations).

Theorem 2.3. The return mapping of the vector field $X$ converges at least on the disc $D(0, R(\rho))$ of radius $R(\rho)=\rho / 2$ where $\rho$ is the unique positive real number such that:

$$
\rho^{d}\left|\lambda_{d}\right|+\ldots+\rho\left|\lambda_{1}\right|=1 .
$$

This last estimation improves (2.12) for small $|\lambda|$ but not for large ones.

Proof. Change of coordinates $(x, y)$ into $(\rho x, \rho y)$ transforms

$$
\begin{gathered}
\dot{x}=y \\
\dot{y}=-x+p(x) y,
\end{gathered}
$$

into

$$
\begin{gathered}
\dot{x}=y \\
\dot{y}=-x+\sum_{i=1}^{d}\left(\lambda_{i} \rho^{i}\right) x^{i} y .
\end{gathered}
$$

This means that any result obtained on the disc $D(0, R)$ for the equation $(2.14 \mathrm{a})$ is valid on the disc $D(0, \rho R)$ for the new equation $\lambda_{i} \mapsto \lambda_{i}^{\prime}=\rho^{i} \lambda_{i}$.

\section{Estimates of the number of complex limit cycles on a fixed neighborhood of the origin}

The generalization of Bautin's approach to complex limit cycles has been done in $([8],[9])$. It yields quite explicit results in the case of homogeneous perturbations of the linear part. The same type of results can be displayed in the case of Liénard equations and this is done in this paragraph.

It is quite interesting to note now that in comparison to the "polynomial Hironaka division theorem" that we need to use in the general situation, we have to check a very easy proposition.

In the case of Liénard equations, $\left(\lambda_{2}, \ldots \lambda_{2 n}\right)$ defines clearly a Hironaka basis (standard basis or Grobner basis). Recall shortly for need of selfconsistency the definition of such a basis.

A total ordering $\leq$ on $N^{D}$ is said to be compatible with the addition if:

i) For all indices $\alpha \in N^{D}, \beta \in N^{D}$, then $\alpha \leq \alpha+\beta$,

ii) For all indices $\alpha^{1}, \alpha^{2}, \beta, \alpha^{1}+\beta \leq \alpha^{2}+\beta$ if and only if $\alpha^{1} \leq \alpha^{2}$.

Definition 3.1. Let $f \in C[\lambda], f \neq 0, f=\sum f_{\alpha} \lambda^{\alpha}$. The largest exponent $\alpha$ so that $f_{\alpha} \neq 0$ is called the privileged exponent of $f$ and is denoted $\exp (f)$. The monomial $\left[f_{\alpha} \lambda^{\alpha}, \alpha=\exp (f)\right]$ is called the initial monomial and denoted $\operatorname{In}(f)$. 
Definition 3.2. Given s polynomials $g_{1}, \ldots, g_{s}$, the associated partition of $N^{D}$ is defined as follows: $\Delta_{1}=\exp \left(g_{1}\right)+N^{D}, \ldots, \Delta_{i}=\exp \left(g_{i}\right)+N^{D}-\bigcup_{j<i} \Delta_{j}, \ldots, \bar{\Delta}=$ $N^{D}-\bigcup_{i=1}^{s} \Delta_{i}$.

Definition 3.3. Let $I$ be an ideal of $C\left[\lambda_{1}, \ldots, \lambda_{D}\right]$. Consider the set:

$$
\exp (I)=(\exp (f), f \in I) .
$$

It can be shown that this set has finitely many extremal points:

$$
\alpha^{1}, \ldots, \alpha^{s} \text {. }
$$

Choose $g_{1}, \ldots, g_{s}$ in the ideal $I$ so that $\exp \left(g_{i}\right)=\alpha^{i}, i=1, \ldots s$. Such a set of polynomials is called a standard basis, Hironaka basis or Grobner basis (of the ideal I relatively to the ordering $\leq$ ).

Proposition 3.4. Let I be the Bautin ideal. Let $f(\lambda)$ be an element of I of degree $k$, there is a decomposition:

$$
f(\lambda)=\sum_{i=1}^{n} \phi_{i}(\lambda) \lambda_{2 i},
$$

with

$$
\operatorname{deg}\left(\phi_{i}\right) \leq \operatorname{deg}(f)-1=k-1
$$

and

$$
\left|\phi_{i}\right| \leq|f| \text {. }
$$

Proof. Consider $f(\lambda)$, collect in front of $\lambda_{2}$ all the monomials which contain $\lambda_{2}$. The difference still belongs to the ideal $I$. Then repeat the process with $\lambda_{4}$, .. . The two majorations are obvious.

Following the techniques of $([8],[9])$, we prove now that the first return mapping belongs to a Bernstein class. It is convenient at this point to change notations and write:

$$
f(\lambda, r)=r+v_{2}(2 \pi) r^{2}+\ldots+v_{k}(2 \pi) r^{k}+\ldots,
$$

for the (converse of the) first return mapping and $f_{k}(\lambda)=v_{k}(2 \pi)$ for its coefficients. Indeed, we consider the analytic extension of the series to the complex domain and denote now $x$ as the complex variable in place of the real variable $r$.

$$
f(\lambda, x)=x+f_{2}(\lambda) x^{2}+\ldots+f_{k}(\lambda) x^{k}+\ldots
$$

Definition 3.5. Let $B$ be an integer, $R>0$ and $c>0$ and $f(z)=\sum_{i \geq 0} a_{i} z^{i}$ be an analytic function on a neighborhood of $0 \in C$. The function $f$ belongs to the Bernstein class $B_{B, R, c}^{2}$ if and only if for all $j \geq B$ :

$$
\left|a_{j}\right| R^{j} \leq \operatorname{cmax}_{i=0, \ldots, B}\left(\left|a_{i}\right| R^{i}\right) .
$$


Proposition 3.6. The analytic series (3.5) belongs to the Bernstein class $B_{(B, R, c)}^{2}$ with:

$$
\begin{gathered}
R=K_{4}(1+|\lambda|), \\
c=n K_{3}^{2} K_{4}^{2 n-1} / c_{2 n+1}^{n} .
\end{gathered}
$$

Proof. Write first the condition for $f_{\lambda}(x)$ to be an $A_{0}$-series as follows:

$$
\begin{gathered}
\operatorname{deg}\left[f_{k}\right] \leq k-1, \\
\left|f_{k}\right| \leq K_{3} K_{4}^{k} .
\end{gathered}
$$

Write next the decomposition:

$$
f_{k}(\lambda)=\sum_{i=1}^{n}\left[\phi_{k, i}(\lambda) \lambda_{2 i}\right] .
$$

This yields:

$$
\left.\left|\phi_{k, i}(\lambda)\right| \leq\left|f_{k}\right|(1+|\lambda|)^{k-2} \leq K_{3} K_{4}^{k-1}(1+|\lambda|)\right]^{k-2} .
$$

This entails:

$$
\mid f_{k}\left(\lambda \leq K_{3} K_{4}^{k-1}(1+|\lambda|)\right]^{k-2}\left(\operatorname{Max}_{i=1, \ldots, n}\left|\lambda_{2 i}\right|\right) .
$$

A more careful analysis of the recurrency relation (cf. 1.19, 1.20) shows that equation (3.5) entails a $n x n$ matrix relation between the vectors $f_{2 j+1}, j=$ $1, \ldots, n$ and $\lambda_{2 i}, i=1, \ldots, n$ of the form:

$$
f_{2 j+1}=\sum_{i=1}^{n}\left[C_{j i}+\Delta_{j i}\right] \lambda_{2 i},
$$

where the matrices $C$ and $\Delta$ are respectively diagonal, with non-zero coefficients $c_{2 i+1}$ defined in (1.20), and nilpotent (upper-triangular). Inverting the matrix relation (3.11) yields:

$$
\left|\lambda_{2 i}\right| \leq\left|(C+\Delta)^{-1}\right| \operatorname{Max}_{i=1, \ldots, n}\left|f_{2 i+1}(\lambda)\right| .
$$

This is completed with the inequalities:

$$
\begin{gathered}
\left|(C+\Delta)^{-1}\right| \leq\left|C^{-1}\right|\left|\left(1+C^{-1} \Delta\right)^{-1}\right|, \\
\leq\left|C^{-1}\right|\left[1+\left|C^{-1} \Delta\right|+\ldots+\left(\left|C^{-1} \Delta\right|\right)^{n-1}\right] .
\end{gathered}
$$

The coefficients of the diagonal matrix $C$ entail (cf. 1.20):

$$
\left|C^{-1}\right|=1 / c_{2 n+1},
$$

This yields:

$$
\left.\left|(C+\Delta)^{-1}\right| \leq\left(1 / c_{2 n+1}\right)^{n} n K_{3} K_{4}^{2 n}(1+|\lambda|)\right]^{2 n+1} .
$$


Equations (3.15), (3.12), (3.9) entail now:

$$
\left|f_{k}(\lambda)\right| R^{k} \leq\left(1 / c_{2 n+1}\right)^{n} n K_{3}^{2}\left[K_{4}(1+|\lambda|)\right]^{2 n-1} \operatorname{Max}_{k=2, \ldots, B}\left(\left|f_{k}(\lambda)\right|\right),
$$

$$
\left|f_{k}(\lambda)\right| R^{k} \leq\left(1 / c_{2 n+1}\right)^{n} n K_{3}^{2}\left[K_{4}(1+|\lambda|)\right]^{4 n} \operatorname{Max}_{j=2, \ldots, B}\left(\left|f_{j}(\lambda)\right|\right) R^{j}
$$

This means that the analytic series (3.3) belongs to the Bernstein class $B_{(B, R, c)}^{2}$ (cf. [8], [9])with:

$$
c=n K_{3}^{2}\left[K_{4}(1+|\lambda|)\right]^{4 n} / c_{2 n+1}^{n}
$$

The proposition 3.2 and the results of $([8],[9])$ now imply the following:

Theorem 3.7. The number of zeros of $f_{\lambda}(x)$ in the disc $D\left(0, R^{\prime}\right)$ is less than $B-1=2 n$ with

$$
\begin{aligned}
R^{\prime} & =c_{2 n+1}^{n} /\left[2^{6 n} n K_{3}^{2}\left[K_{4}(1+|\lambda|)\right]^{4 n+1},\right. \\
R^{\prime} & =c_{2 n+1}^{n} /\left[2^{10 n-1} n \pi^{2}(1+|\lambda|)^{4 n+1} .\right.
\end{aligned}
$$

For small values of $|\lambda|$, this estimate can be improved with the same scaling argument as used in the previous paragraph as follows:

Theorem 3.8. The vector field $X$ has less than $2 n$ complex limit cycles on the disc $D\left(0, R^{\prime}(\rho)\right)$ of radius

$$
R^{\prime}(\rho)=\rho c_{2 n+1}^{n} /\left[\pi^{2} n 2^{14 n}\right]
$$

where $\rho$ is the unique positive real number such that:

$$
\rho^{d}\left|\lambda_{d}\right|+\ldots+\rho\left|\lambda_{1}\right|=1 \text {. }
$$

\section{Acknowledgements}

The author thanks M. Caubergh for a careful reading of the manuscript. This article was completed during a visit to CRM of Montréal. The author is grateful to L. Belair, C. Rousseau and D. Schlomiuck for their invitation and to V. Kaloshin and S. Yakovenko for useful comments related to the presentation of the results.

\section{References}

[1] T. R. Blows, N. G. Lloyd, The number of small-amplitude limit cycles of Liénard equations, Math. Proc. Cambridge Philos. Soc. 95 (1984), no. 2, 359-366.

[2] M. Briskin, J.-P. Francoise, Y. Yomdin, The Bautin ideal of the Abel Equation, Nonlinearity 11 (1998), no. 3, 431-443.

[3] _ Center conditions, composition of polynomials and moments on algebraic curves, Ergodic Theory Dynam. Systems 19 (1999), no. 5, 1201-1220.

[4] M. Briskin, Y. Yomdin, Algebraic families of analytic functions. I, J. Differential Equations. 136 (1997), no. 2, 248-267.

[5] C. J. Christopher, N. G. Lloyd, Small-amplitude limit cycles in Liénard systems, Nonlinear Differential Equations Appl. 3 (1996), no. 2, 183-190. 
[6] G. Dangelmayr, J. Guckenheimer, On a four parameter family of planar vector fields, Arch. Rational Mech. Anal. 97 (1987), no. 4, 321-352.

[7] F. Dumortier, C. Rousseau, Cubic Liénard equations with linear damping, Nonlinearity 3 (1990), no. 4, 1015-1039.

[8] J.-P. Francoise, Y. Yomdin, Bernstein inequalities and applications to analytic geometry and differential equations, J. Funct. Anal. 146 (1997), no. 1, 185-205.

[9] _ Projection of analytic sets and Bernstein inequalities, Singularities SymposiumŁojasiewicz 70 (Kraków, 1996; Warsaw, 1996), 103-108, Banach Center Publ., 44, Polish Acad. Sci., Warsaw, 1998.

[10] M. Hirsch, S. Smale, Differential equations, dynamical systems and linear algebra, Pure and Applied Mathematics, Vol. 60. Academic Press, New York-London, 1974.

[11] A. Gasull, J. Torregrosa, Small-amplitude limit cycles in Liénard systems via multiplicity, J. Differential Equations 159 (1999), no. 1, 186-211.

[12] A. Lins neto, W. de Melo, C. C. Pugh, On Liénard equations, Geometry and topology (Proc. III Latin Amer. School of Math., Inst. Mat. Pura Aplicada CNPq, Rio de Janeiro, 1976), 335-357. Lecture Notes in Math., Vol. 597, Springer, Berlin, 1977.

[13] A. I. Khibnik, B. Krauskopf, C. Rousseau, Global study of a family of cubic Liénard equations, Nonlinearity 11 (1998), no. 6, 1505-1519.

[14] N. G. Lloyd, Liénard systems with several limit cycles, Math. Proc. Cambridge Philos. Soc. 102 (1987), no. 3, 565-572.

[15] N. G. Lloyd, S. Lynch, Small-amplitude limit cycles of certain Liénard systems, Proc. Roy. Soc. London Ser. A 418 (1988), no. 1854, 199-208.

[16] S. Lynch, Small-amplitude limit cycles of Liénard systems, Calcolo 27 (1990), no. 1-2, 1-32, (1991).

[17] Liénard systems and the second part of Hilbert's sixteenth problem, Nonlinear Anal. 30 (1997), no. 3, 1395-1403.

[18] S. Malo, Rigorous computer verification of planar vector field structure, Ph. D. thesis, Cornell University, 1994.

[19] S. Smale, Dynamics retrospective: great problems, attempts that failed, Nonlinear science: the next decade (Los Alamos, NM, 1990). Phys. D 51 (1991), no. 1-3, 267-273.

[20] _ Mathematical problems for the next century, Math. Intelligencer, 20 (1998), no. 2, $7-15$.

Université de Paris Vi, Laboratoire GSiB, Site Chevaleret, Bureau 8C20, 175 Rue de Chevaleret, 75013 Paris, France.

E-mail address: jpf@ccr.jussieu.fr 